Vol. 3, N².1., p. 260-283, junio, 2019

Recibido: 05-04-2019 /Aceptado: 08-05-2019/ Publicado: 07-06-2019

\title{
Sistema de gestión de costos ABC/ABM para la industria de textiles. Caso empresa Vatex.
}

\section{Activity-Based Costing System ABC/ABM for Textile Industries. Case Vatex \\ Company}

Henry David González Montaño. ${ }^{1}$, Cecilia Ivonne Narváez Zurita. ${ }^{2}$, Mónica Alexandra Lituma Yascaribay. ${ }^{3}$ \& Juan Carlos Erazo Álvarez. ${ }^{4}$

DOI: https://doi.org/10.33262/visionariodigital.v3i2.1.555

\begin{abstract}
.
Currently, globalization and economic development have boosted companies to assess the efficiency and effectiveness of their operations to compete successfully. Therefore, there is a special requirement to determine their production costs, revenues, and profits, so that they need means to gather relevant and reliable information. In this regard, the Activity-Based Costing System ABC/ABM is an essential tool to determine the actual production cost through an analysis of processes, activities, and cost drivers.

Consequently, this research has evaluated the costing methods to conclude that the Activity-Based Costing System ABC/ABM is the most accurate approach to establish the production costs of the Company Vatex, located in the city of Cuenca-Ecuador. The study was developed through a mixed method using a descriptive-explanatory approach. For gathering information, there have been used surveys and interviews with the staff of the production area, accounting, logistics, and management; among the leading results obtained is the costing of two company production lines (T-shirts and pants).
\end{abstract}

\footnotetext{
${ }^{1}$ Universidad Católica de Cuenca, Posgradista Maestría en Contabilidad y Auditoría. Cuenca, Ecuador.hdgonzalezm173@psg.ucacue.edu.ec

${ }^{2}$ Universidad Católica de Cuenca, Subdirección de Posgrado, Cuenca, Ecuador, inarvaez@ucacue.edu.ec ${ }^{3}$ Universidad Católica de Cuenca, Facultad de Administración. Cuenca, Ecuador. mlitumay@ucacue.edu.ec ${ }^{4}$ Universidad Católica de Cuenca, Subdirección de Posgrado, Cuenca, Ecuador, jcerazo@ucacue.edu.ec
} 
Keywords: Activity-Based Costing System, Cost Drivers, Activities, Process, Profitability.

\section{Resumen.}

En la actualidad la globalización y el desarrollo económico, impulsan a las empresas a evaluar la eficiencia y eficacia de sus operaciones para lograr competir con éxito, y para ello requieren establecer con precisión sus costos de producción, ingresos y utilidades, es así que necesitan herramientas que les permitan obtener información relevante y confiable. En este sentido, el sistema de costos basado en actividades $\mathrm{ABC} / \mathrm{ABM}$ se constituye en una herramienta óptima para determinar el costo real de producción mediante el análisis de procesos, actividades e inductores del costo.

En tal razón, en la presente investigación se realizó una revisión de los métodos de costeo, llegando a la conclusión que el sistema $\mathrm{ABC} / \mathrm{ABM}$ es el método más adecuado para determinar los costos de producción en la empresa Vatex, localizada en la ciudad de Cuenca, Ecuador. La investigación se desarrolló bajo un enfoque mixto, con un alcance descriptivo - explicativo, con orientación transversal. Los instrumentos aplicados fueron la encuesta y la entrevista dirigida al personal de las áreas de producción, contabilidad, logística y a la gerencia, entre los principales resultados se obtuvo el costeo de dos líneas de producción de la empresa (camisetas y pantalones).

Palabras claves: Costos $\mathrm{ABC} / \mathrm{ABM}$, inductores, actividades, procesos, rentabilidad.

\section{Introducción.}

Los costos se encargan de recopilar la información de la transformación de insumos en productos o servicios para que estos puedan llegar a un sector en específico. Considerando como tales, todos aquellos insumos que intervienen en la producción y puedan ser recuperados en su posterior venta, entendiendo que los elementos que los conforman son materia prima, mano de obra e insumos. (Cuervo, Osorio y Duque, 2013). Rincon (2012) manifiesta que los costos son iversiones que se realizan con el objetivo de obtener un rédito o beneficio a futuro, en este caso, al momento de reconocer el valor o costo que se le da a cada actividad resulta ser la inversión inicial, de esa manera se pueden establecer el ingreso para obtener el beneficio esperado.

La contabilidad de costos tiene 3 elementos fundamentales, los cuáles intervienen directamente en la producción de un bien o un servicio, estos son: materiales directos, mano de obra directa y gastos indirectos de fabricación. 
Materia prima: al hablar de materia prima se debe considerar a todos aquellos bienes e insumos que de manera directa ingresan al proceso de producción del bien o servicio. Un claro ejemplo en la industria textil, sería en gran cantidad la tela, botones, broches, cierres, cordón, entretela; estos materiales llegas a formar la parte principal del producto. Arrellano, Quishpe, Ayaviri y Escobar (2017) definen a la materia prima como los principales recursos o elementos físicos que son imprescindibles y se consumen durante el proceso de fabricación de un producto, estos se transforman en bienes terminados mediante la manipulación, de la mano de obra y los costos indirectos de fabricación. Se debe considerar que el uso de los materiales tiene una relación directa en unidades con la cantidad que se produce.

Mano de obra: se refiere al personal debidamente capacitado para realizar actividades directas con el producto, dependiendo del campo en el que se aplique, comúnmente se les conoce como operarios u obreros. Es el esfuerzo físico o mental empleado en la transformación de la materia prima en un producto. Es facultad del contador o departamento contable, establecer la diferencia entre el personal que labora en las distintas áreas, sean estas producción, administración o ventas, dependiendo la naturaleza y alcance de cada empresa, esto a la final conformará individualmente y por departamento la fuerza de la mano de obra (ibídem).

Costos o gastos indirectos de fabricación: son todos aquellos materiales, insumos o servicios que forman parte como complemento para la elaboración del producto en sí, y que no conforman la parte principal del bien o servicio. Cómo ejemplos se tiene los suministros eléctricos, combustibles, agua, desgaste de equipos (depreciaciones), herramientas, arrendamiento de espacios (Eras, Burgos, \& Lalangui, 2016). Por su parte, Arrellano et al., (2017) señalan que los gastos indirectos de fabricación son aquellos valores que no son considerados como parte final del producto, pero si indispensables, entre estos: los materiales indirectos, mano de obra indirecta, y todos los demás elementos que no son fácilmente identificables directamente en el producto. Como componentes se pueden clasificar de la siguiente manera: materia prima indirecta, mano de obra indirecta, costos indirectos de fabricación, combustibles y lubricantes, depreciaciones de planta, maquinaria y herramientas, energía eléctrica, agua potable, teléfono, internet, seguro, impuestos, inmobiliarios y, transporte de materiales

Para establecer el sistema de costos es necesario enfocarse en los siguientes aspectos: a) analizar e identificar las diferentes actividades de la empresa, b) establecer el valor monetario y resultado de cada actividad, c) rastrear el costo de las actividades y d) evaluar la efectividad y eficiencia de las actividades. Los diferentes sistemas de costos se pueden definir de acuerdo a las necesidades de la entidad o en base a los procesos previamente establecidos por la alta gerencia y el control que se ejerza en la organización. En la tabla 1 se detalla las características de los sistemas tradicionales: 
Tabla 1. Sistemas de costos

\begin{tabular}{|c|c|c|}
\hline Sistema & Definición & Características \\
\hline $\begin{array}{l}\text { Por órdenes de } \\
\text { fabricación o } \\
\text { producción }\end{array}$ & $\begin{array}{lr}\text { Es un sistema que } \\
\text { registra y acumula } \\
\text { costos de acuerdo a } \\
\text { una orden de } \\
\text { producción } r \text { o } \\
\text { fabricación, aplicables } \\
\text { en empresas } \\
\text { productoras } \quad \text { de } \\
\text { servicios y bienes. }\end{array}$ & $\begin{array}{l}\text { - Se trabaja por especificaciones } \\
\text { dadas por el cliente. } \\
\text { - Se acumulan los costos por } \\
\text { cada orden solicitada por el } \\
\text { cliente. } \\
\text { - El costo unitario se calcula al } \\
\text { terminar el trabajo. } \\
\text { - Trabaja con hojas de costos } \\
\text { acumulativas. }\end{array}$ \\
\hline Por procesos & $\begin{array}{l}\text { Registra y acumula los } \\
\text { costos de acuerdo a la } \\
\text { producción de la } \\
\text { empresa, es decir, la } \\
\text { magnitud de } \\
\text { producción es alta y } \\
\text { continua. Se sigue una } \\
\text { línea de proceso a } \\
\text { proceso y el último } \\
\text { acumulará los costos } \\
\text { totales. }\end{array}$ & $\begin{array}{l}\text { - Producción continua y masiva. } \\
\text { - Productos homogéneos. } \\
\text { - Los costos se acumulan por } \\
\text { centros de costo. } \\
\text { - El costo unitario se calcula para } \\
\text { un departamento. } \\
\text { - Cada departamento o centro de } \\
\text { costos emite su informe de } \\
\text { costos incurridos. }\end{array}$ \\
\hline
\end{tabular}

En este sentido, Molina y Morillo (2000), mencionan que el objetivo principal de un sistema de costos se basa en la capacidad de establecer el costo que se genera en la prestación de un servicio o la realización de un producto, a través de los recursos disponibles para su ejecución, sin dejar de lado el análisis del rendimiento, permitiendo que se creen diversas formas de aplicaciones que puedan ayudar a incrementar sus beneficios.

Los sistemas de costeo tradicional no permiten conocer los inductores del costo o la confiabilidad de los costos unitarios, esto debido a que no identifican procesos, actividades o áreas de la producción. En cambio, el sistema de costeo ABC evalúa las actividades, identificando las áreas donde se generan los costos y permitiendo generar información confiable, conocer las actividades que generan valor o actividades que son innecesarias. 


\section{Gestión basada en costos ABC/ABM}

El costo basado en $\mathrm{ABC} / \mathrm{ABM}$ debe ser tratado de manera simultánea. La gestión de costos tiene dos componentes importantes que conforman el costeo: ABC y, ABM. Kaplan y Anderson (2008) manifiestan que el sistema de costos ABC empieza a aplicarse en la década de 1980 como respuesta a que los sistemas de costos tradicionales, no reflejaban la realidad económica que se vivía en las empresas, principalmente debido a que se distorsionaba la información sobre la rentabilidad generada. En este contexto, Pérez y Tapanes (2009), señalan que "el Sistema de costo $\mathrm{ABC}$, se basa en la agrupación en centros de costos que conforman una secuencia de valor de los productos y servicios de la actividad productiva de la empresa." (p.16). Teniendo en cuenta que una actividad emplea recursos con la finalidad de obtener un producto, el análisis se basa en actividades por departamentos de costos, con la finalidad de determinar el consumo de los recursos necesarios para la fabricación del producto final, permitiendo establecer la rentabilidad de los mismos (Adame, 2000).

El ABM es una disciplina que se centra en la gestión de las actividades como vía para mejorar el valor recibido por los clientes y el beneficio alcanzado al proporcionar dicho valor. Esta disciplina incluye el análisis de lo que genera costes, el análisis de las actividades desarrolladas por la empresa y la medida del rendimiento (Navarro, 1995). El ABM tiene por objeto definir los procesos y actividades que se desarrollan dentro del proceso productivo, los mismos pueden estar establecidos en tres posiciones: 1) procesos estratégicos que se orientan a las actividades a nivel estratégico, 2) procesos operativos que se enfocan directamente a las operativas o productivas, 3 ) procesos de apoyo que se enfocan a actividades adicionales como: limpieza, mantenimiento, reparación, o en otros casos los encargados de proveer de materiales y recursos. Una vez que se realiza este proceso se analizan las actividades y su respectiva clasificación y jerarquización en primarias y secundarias, identificando cuáles son las que generan valor para la empresa.

Para determinar la gestión de costos ABC/ABM, es necesario conocer el significado de los términos que se utilizan para la aplicación, los cuáles se detallan a continuación:

a. Actividades: son los elementos que una empresa puede controlar, una actividad implica el uso de recursos sean estos materiales, insumos, recurso humano, y la forma en cómo son empleados para el cumplimiento de los objetivos establecidos previamente (Adame, 2000). Es así que, las actividades son consideradas el componente más importante para el manejo de los costos $\mathrm{ABC}$ dentro de una organización.

$b$. Recursos: se puede considerar como recursos a todos aquellos insumos o medios, sean estos materiales, personal, equipos, maquinaria, instalaciones, con los cuales se cuenta para poder desarrollar cualquier actividad. Según Cuervo, Osorio y Duque (2013), estos recursos se pueden clasificar en: a) contables, cuando se encuentran debidamente 
registrados en la contabilidad y, b) no contables, aquellos que no se pueden incluir dentro de la contabilidad.

c. Conductor o driver: según Adame (2000), existen dos tipos de drivers: a) los inductores de actividades, que miden el costo de una actividad $\mathrm{y}, \mathrm{b}$ ) los inductores de recursos, que se enfocan en la medición de los recursos empleados por las activadades, teniendo en cuenta que estos inductores al mismo tiempo son el enlace entre los recursos y las actividades. El driver de costo es el elemento o factor que da entrada al costo, entre ellos todos los aspectos relevantes en cuanto al uso y consumo de todos los recursos que se emplean para la producción. Es muy importante conocer la existencia de diferentes tipos de inductores, entre ellos se pueden mencionar: a) inductores de operaciones, son los más simples y miden la frecuencia con que se desarrolla la actividad, b) inductores de duración, son los que tienen en cuenta el tiempo que demanda la actividad y, c) inductores de intensidad, que asumen una imputación directa, son los más precisos pero los más caros debido al análisis que debe hacerse acerca del uso de la actividad por el producto.

En base a lo expuesto anteriormente, Cano (2013) menciona que el costeo ABC es una herramienta que facilita la planeación de las operaciones y las diferentes actividades que generan valor; así mismo, ayuda a suprimir aquellas actividades que no son útiles o que retrasan los procesos, continuyendo a mejorarlos para aportar viablemente al cumplimiento de los objetivos preestablecidos. Uno de los objetivos básicos del costeo ABC es gestionar costos en las diferentes actividades que agregan valor, y buscar reducir los costos eliminando actividades que generan poco valor, rediseñando o proponiendo nuevas alternativas que aceleren y mejoren la eficiencia de los diferentes procesos que se dan en la producción del bien o servicio.

La capacitación correcta del personal permite que los aportes positivos de este sistema sean evidentes. Dichas capacitaciones incluyen el conocimiento para aplicar esta herramienta, partiendo de la identificación de los diferentes factores que permitan mantener y mejorar la fiabilidad de la información para la toma de decisiones estratégicas. Para tener un mejor entendimiento de los beneficios se deben aplicar ciertos pasos que permitan dar razonabilidad al sistema, entre estos: adquirir la capacidad de manejar el sistema, socializar sus facilidades y ventajas, ser claro, entendible y conciso para los usuarios, levantar un análisis general de la empresa y, crear un vínculo entre el giro comercial y el análisis de costos.

Para una adecuada implementación del costeo ABC/ ABM, Horngren, Datar y Rajan (2012) plantean los siguientes pasos a) establecer los productos objetos del costo, b) definir los costos directos, c) seleccionar las actividades y la base de asignación de costos indirectos de fabricación, d) identificar los costos indirectos asociados con la base de asignación, e) calcular la tasa por unidad y, f) calcular los costos indirectos asignados al producto. Por otra parte, para Cano (2013), resulta indispensable desagregar ciertos pasos para que puedan ser 
entendidos, situándose entre el punto c y d, que son la determinación de generadores o los denominados driver, siendo capaces de responder a las situaciones causa-efecto de las actividades; y de igual manera, la reclasificacion de actividades, identificando actividades similares que pueden ser fácilmente unificadas para reducir procesos y costos.

La aplicación de costos, puede centrarse en la formulación de las fases de implementación de un sistema o modelo de gestión de costos, tal como se evidencia en la figura 1.

Figura 1. Modelo gestión de costos.

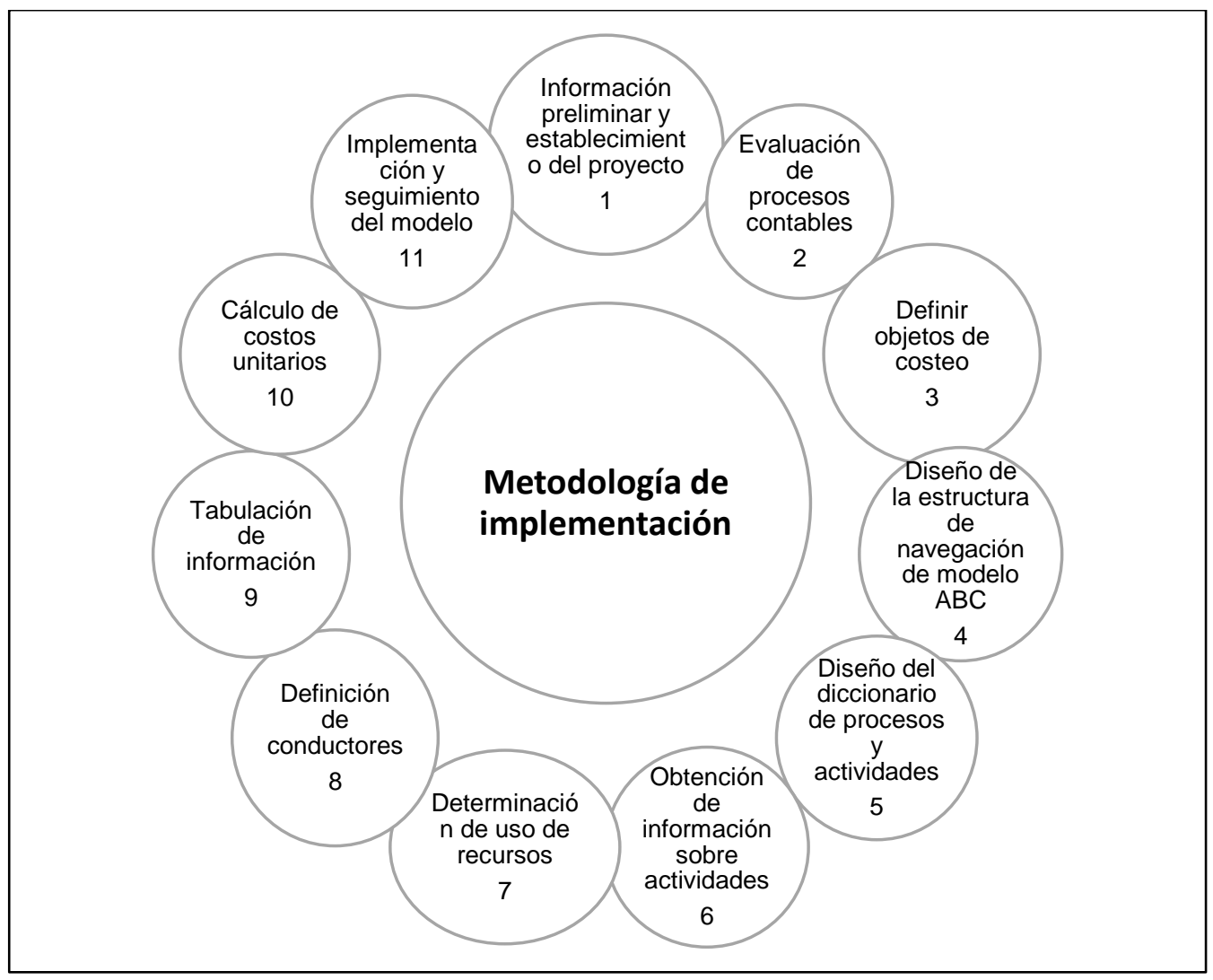

Fuente: (Cuervo, Osorio y Duque, 2013)

El modelo presentado por Cuervo et al. (2013), muestra una metodología que incluye los pasos desde la idea hasta el seguimiento del modelo a implementar. Cada paso incorpora una serie de procedimientos que se deben emplear para tener una idea clara y concreta de lo que se va a aplicar y de cómo se va a aplicar dentro de una organización, tal como se detalla a continuación:

a) Dentro de la información preliminar y el establecimiento del proyecto, se contempla el vender la idea de manera estratégica a la organización. Además, como es un proyecto, su primer paso considera el conocimiento general de la empresa en materia 
de controles y procesos que utilizan para las diferentes actividades empleadas para producción o control.

b) Se considera los procesos que forman parte de los registros contables, aspectos con respecto a su valoración, el uso, descargo y todas las operaciones que conlleve su manejo, esto incluye al personal que se encuentra a cargo con las diferentes actividades que realiza, tomando en cuenta las medidas de control que se aplican y posibles filtros de información hacia la contabilidad.

c) La determinación del objeto de costeo se refiere básicamente al producto o servicio que va a ser razón de estudio de costos.

d) Al tener definido el objeto del costo, se analiza las actividades que contempla la producción, pudiendo así definir paso a paso los procesos que se siguen para la producción del bien o servicio.

e) Una vez que contamos con la estructura, se define de forma detallada las actividades que se desarrollan en cada proceso.

f) En las actividades intervienen factores como tiempo, mano de obra y recursos, los cuáles deben tener su levantamiento y deben ser plasmados correctamente para poder seguir con el proceso de forma adecuada.

g) Se determina el uso de los recursos, los mismos que pueden ser humanos o económicos.

h) La determinación de conductores se orienta directamente a la actividad a la cual se va a aplicar. Un error frecuente es asignar gastos de fabricación indirectos a los productos sin haber utilizado una técnica o herramienta. A diferencia del tradicional, el $\mathrm{ABC} / \mathrm{ABM}$ considera los diferentes eventos y diferencias que se pueden generar entre los productos y servicios (Adame, 2000).

i) Con la información de los costos, se procede a calcularlos unitariamente, esto ayuda a establecer estrategias para precio de venta al consumidor.

j) Una vez ya definidos todos los parámetros, se puede implementar el modelo resultante de la investigación. El seguimiento de esto conlleva a la evaluación del desempeño del modelo, para lo cual se establecen los responsables de cada actividad, y luego de cada informe se podrá mejorar el modelo ya establecido afinándolo o incorporando nuevos procesos que conlleven al cumplimiento del objetivo estratégico de la entidad.

Las empresas buscan mejorar su rentabilidad, a través del mejoramiento de las ganancias, estos resultados se obtienen al momento de aplicar la gestión de costos, con la reducción de actividades innecesarias, innovaciones tecnológicas, y capacitaciones. Estos resultados son evaluados por los inversionistas que no solo se enfocan en datos cualitativos, sino también de las utilidades que les pueden generar otras inversiones. (Morillo, 2001) 
Debido a la globalización y el desarrollo económico, las empresas se han visto obligadas a evaluar la eficiencia y eficacia de sus operaciones. La construcción de indicadores ayuda a identificar qué puntos hay que fortalecer y poder tomar decisiones acertadas en base a los resultados obtenidos, a la vez permite que surjan nuevas ideas de cómo incrementar y obtener los objetivos que se promueven en la alta gerencia (Aragon y Rubio, 2006; Santiesteban, Fuentes, Leyva, Lozada y Cantero, 2011; Cantero y Leyva , 2016).

La inversión es considerada como el aporte que se realiza con la finalidad de poder recuperar aquellos valores a corto o a largo plazo; dependiendo del fin con que se utilice, estos pueden generar un beneficio a futuro, por esta razón no puede ser considerado directamente como un gasto debido a las características de retorno que definen a la inversión.

La rentabilidad en cambio se define como el beneficio obtenido por las inversiones que se realizan para la obtención de los diferentes productos o servicios que puede producir una entidad, en otras palabras, es el resultado que se obtiene como consecuencia de las decisiones que previamente se han tomado para el giro normal de las actividades de la empresa. Constituye una medida del rendimiento de la colocación de un capital en un tiempo determinado, utilizando para los análisis diferentes razones que permitan establecer la eficiencia y poner atención en los resultados que se obtienen de las acciones previamente dictadas para la ejecución de procesos. (Sanchez, 2002). La rentabilidad es uno entre varios métodos que se emplean para medir el rendimiento de cada entidad, dependiendo de la aplicación que se realice, estos se basarán en las ventas, en las inversiones, y en lo que posee cada empresa, es decir los activos (Robles, 2012). Bajo esta perspectiva, existen varios enfoques para poder definir directamente la rentabilidad, estos se pueden dar desde el punto de vista financiero, contable o marketing, estableciendo como parámetro el análisis contable, se debe tener en cuenta que hay una correlación entre la rentabilidad y el riesgo, un análisis entre las dos variables permite tener una visión clara de los resultados obtenidos en determinado ciclo contable o económico.

En base a lo expuesto, el análisis se realiza considerando la rentabilidad o ganancia que se obtiene de cada ejercicio, comparando con diferentes factores que posee cada empresa, dando una guía o un acercamiento a un resultado entendible para los interesados, sean estos la alta gerencia, socios, accionistas, o instituciones externas. Dependiendo de la necesidad del análisis, se puede considerar impuestos o así mismo costo de financiamiento, pero que de cualquier manera ayudan a clarificar los resultados que se obtienen.

Sanchez (2002) establece que el análisis de la rentabilidad se basa en la correlación de este factor con el riesgo, ya que al tomar un riesgo se tiene como resultado la rentabilidad. Al tener esto, se pueden definir tres aspectos importantes que nacen de los beneficios: la rentabilidad, la solvencia y la estructura financiera de la entidad, lo cuál se busca aclarar y 
establecer en cada análisis. Maldonado (2003) menciona que la contabilidad es un aspecto importante para la toma de decisiones, reflexiona sobre la información que se genera desde aquí para el respectivo análisis. Chacón (2007) coincide que los balances y estados que se emiten por parte de la contabilidad son escenciales para el análisis respectivo, siendo fácil ubicar lo datos que se necesitan para estructurar los indicadores.

De acuerdo a la naturaleza de las actividades y giro empresarial, se plantean como base del análisis los siguientes enfoques, encontrándose la relación riesgo y rentabilidad entrelazadas: a) análisis de la rentabilidad, b) análisis de la solvencia y, c) análisis de la estructura financiera. (Santiesteban et al. 2011).

En la descomposición del estado de resultados, los costos tienden a ser uno de sus componentes, al ser analizado dentro de la rentabilidad tiende a dar un resultado del consumo de los recursos para la producción, en otras palabras, es el resultado del consumo de la inversión realizada, lo que indica que en el uso de las razones se encuentra los costos incluidos. El análisis de razones puede incluir valores directamente con resultados totales, pero al mismo tiempo se pueden segregar para ser usados en análisis más profundos como el del margen de rentabilidad con respecto a los costos de producción, de esa manera se puede establecer un análisis por componentes del estado de resultados, siendo útil para ver reflejados los resultados de las decisiones tomadas por la alta gerencia.

La finalidad del análisis financiero de una empresa, radica en la identificación de la solvencia o de la debilidad de la organización en base a resultados obtenidos, adicionalmente, estas pueden contener información importante para cada uno de los usuarios, recordando que todos tienen una visión diferente para interpretar los resultados. Shim y Siegel (1988) establecen algunas limitaciones sobre el uso de estos ratios:

- No se pueden comparar los resultados con otras empresas, si no se encuentran con el mismo giro del negocio.

- Las prácticas contables difieren de empresa a empresa, es decir cada contador tiende a registrar los procesos según su criterio, por lo que tampoco resulta útil relacionarla con otras entidades.

- La información se basa en datos históricos propios de la entidad.

- La confiabilidad de las razones depenerá de la veracidad con la que sean calculados.

- Las razones son un resultado general, no reflejan los movimientos de grupos particulares.

- Los indicadores son estáticos, no tienden a ser predictivos.

El análisis de la rentabilidad no solo implica un indicador interno para la empresa; los inversionistas se basan en estos resultados para tomar el riesgo de ser parte de un negocio, 
tal es el caso del costo promedio ponderado o weighted average cost of capital (WACC), que básicamente es una tasa de descuento que evalúa el riesgo económico y financiero de inversiones que ya se encuentran realizadas, estableciendo un porcentaje que se convierte en la rentabilidad para atraer inversores a proyectos.

La fórmula para el cálculo del WACC es la siguiente:

$$
\text { WACC }=\frac{\text { Df }}{\text { Rt }} \times \text { Cdf }+\frac{F p}{R t} \times \text { Cfp }
$$

En dónde:

Df: Deudas financieras

Cdf: Costo de deudas financieras

Fp: Fondos propios

Cfp: Costos de fondos propios

Rt: Recursos totales

\section{Metodología.}

La presente investigación tuvo un enfoque mixto ya que mediante la aplicación de procesos sistémicos y críticos se recolectaron y analizaron datos cuantitativos y cualitativos, posteriormente se logró la integración y discusión conjunta de la información para realizar inferencias que permitieron un entendimiento claro de las dificultades que atraviesa la empresa Vatex para la determinación real de los costos de producción. El estudio se desarrolló bajo un alcance descriptivo - explicativo, ya que la primera fase se describió con precisión las variables de estudio, en tanto que, en la segunda fase a partir de la recopilación de la información se explicaron en detalle las condiciones en las que se manifiesta el problema de investigación en la unidad de análisis. La finalidad de la investigación fue transversal, debido a que se analizaron los procesos actuales y pasados, pero la aplicación de los instrumentos de investigación para el levantamiento de información se hizo un solo momento de tiempo.

Los métodos emppleados fueron: el histório - lógico que permitió el análisis de la evolución de la contabilidad de costos y de los sistemas de costeo. El método inductivo - deductivo permitió establecer estrategias de razonamiento lógico para identificar premisas particulares sobre la gestión de costos ABC/ABM y la rentabilidad y así llegar a conclusiones generales y específicas. El método analítico - sintético posibilitó el análisis de la literatura especializada mediante la descomposicion del objeto de estudio en cada una sus partes, para finalmente 
presentar de forma resumida bajo una mirada holística e integral la gestión de costos. Las técnicas utilizadas en la recolección de datos fueron la encuesta, entrevista y revisión documental, para ello se consideró como universo de estudio a 9 personas que integran las áreas de contabilidad, logística, producción, y gerenciales: y 4 órdenes de producción correspondientes al mes de abril del 2019.

\section{Resultados.}

A continuación, se presentan los principales resultados obtenidos en la fase de diagnóstico:

Informes gerenciales. - se evidenció que la empresa no utiliza informes gerenciales para la toma de decisiones, puesto que esta información es preparada únicamente con la finalidad de entregar a entidades bancarias cuando solicitan créditos para financiar operaciones.

Análisis de utilidades. - ocasionalmente se realizan análisis de las utilidades, empleados básicamente con fines tributarios. Los análisis esporádicos realizados se basan en el costo del producto generado por el área producción y su precio de venta individual.

Estados financieros. - los estados financieros no presentan los resultados que se esperan por los socios, pero son fácilmente manejables y sus cuentas identificables. Brindan la información que se necesita para análisis y toma de decisiones.

Informes de logística. - los informes son empleados para verificar la rotación de mercadería, así mismo reflejan las solicitudes de producto realizada por cada tienda; garantizan que los niveles de stock en tiendas no sobrepasen su capacidad máxima.

Análisis de rentabilidad. - para mejorar la rentabilidad establecen metas por tienda que deben ser cumplidas con un porcentaje de cumplimiento por el personal de ventas, estas metas tratan de cubrir los gastos generales de la empresa.

Retorno de la inversión. - la empresa no realiza análisis de retorno de la inversión, debido a que evalúan la rotación de la mercadería para medir su rentabilidad.

Grado de identificación de las actividades. - se encuentran identificadas, cada área tiene definidas las actividades que debe realizar.

Grado de identificación de los elementos del costo. - se evidenció que la empresa no tiene conocimiento de sus costos de producción, su método empírico de asignación de costos no refleja el costo real, a excepción de la materia prima. 
Grado de distribución de recursos. - no cuentan con un sistema de costos que permita realizar un análisis de asignación de costos.

\section{Propuesta.}

A partir de los resultados obtenidos en el diagnóstico se propone un modelo de costeo basado en actividades para la empresa Vatex (figura 2).

Figura 2. Esquema de la propuesta

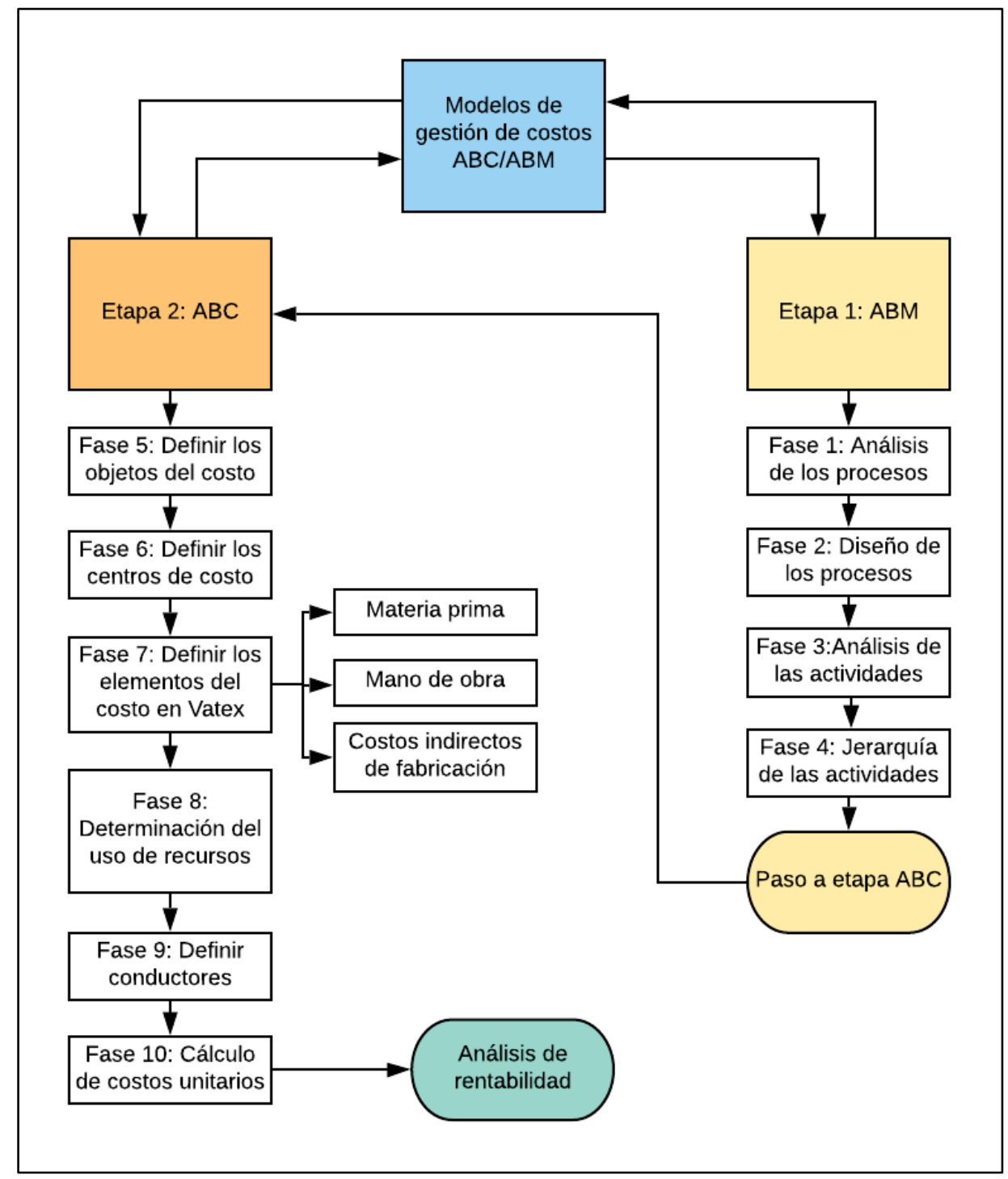

Fuente. Vatex

En este apartado se tratarán los procesos que se desarrollan en la industria Vatex, los cuales se han estructurado en tres niveles: a) procesos estratégicos, establecidos dentro del marco 
administrativo de la empresa, b) procesos operativos, conformados por las áreas encargadas de la gestión operativa y, c) procesos de apoyo, integrados por los diferentes departamentos que no tienen contacto directo con el área operativa. Para garantizar que los procesos sean eficientes, se propone una reorganización de los departamentos que conforman la entidad, tal como se detalla en el organigrama estructural que se muestra a continuación:

Figura 3. Organigrama estructural de la empresa Vatex

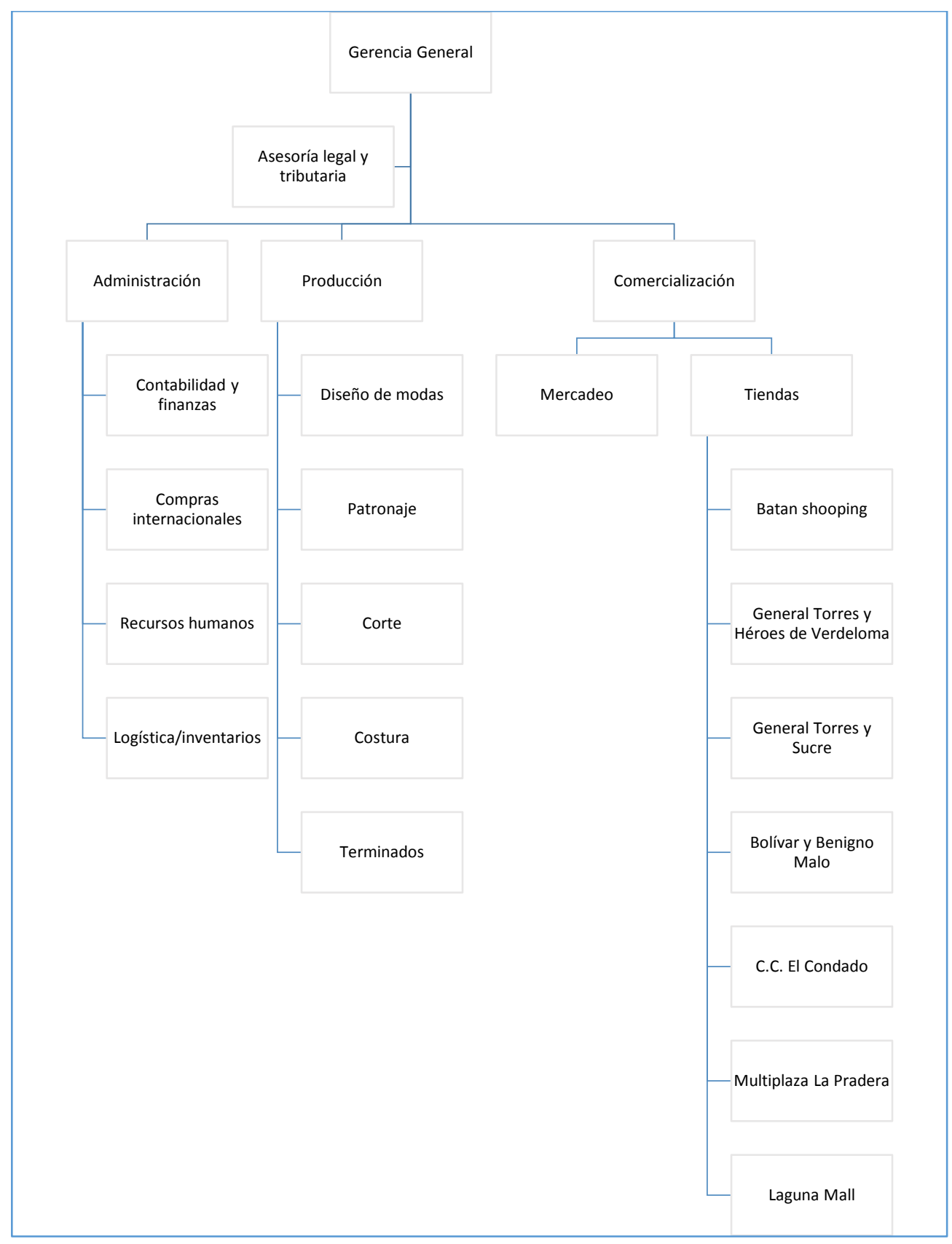

Fuente. Vatex 
Una vez propuesto el organigrama se procede a definir las actividades de cada departamento:

Tabla 2. Actividades de las áreas de la empresa VATEX

\begin{tabular}{ll}
\hline Área & Actividades \\
\hline Gerencia general & Definición y seguimiento de la estrategia \\
Asesoría legal y tributaria & Gestión de litigios \\
Contabilidad y finanzas & Gestión contable \\
& Gestión financiera \\
Compras internacionales & Gestión de importaciones \\
Recursos humanos & Reclutamiento de personal \\
& Evaluación de personal \\
Logística/inventario & Cumplimiento de reglamento de salud y seguridad ocupacional \\
Diseño de modas & Distribución de mercadería \\
Patronaje & Gestión de inventarios \\
Corte & Gestión de diseños en tendencias \\
Costura & Elaboración de patrones \\
Terminados & Corte por patronaje \\
Mercadeo & Unión de patrones \\
Tiendas & Colocación de botones, broches y planchado de prendas \\
\hline
\end{tabular}

Fuente. Vatex

Con esta información se procede a clasificar los procesos en sus tres niveles: estratégicos, operativos y de apoyo. 
Figura 4. Mapeo de los procesos de la industria VATEX

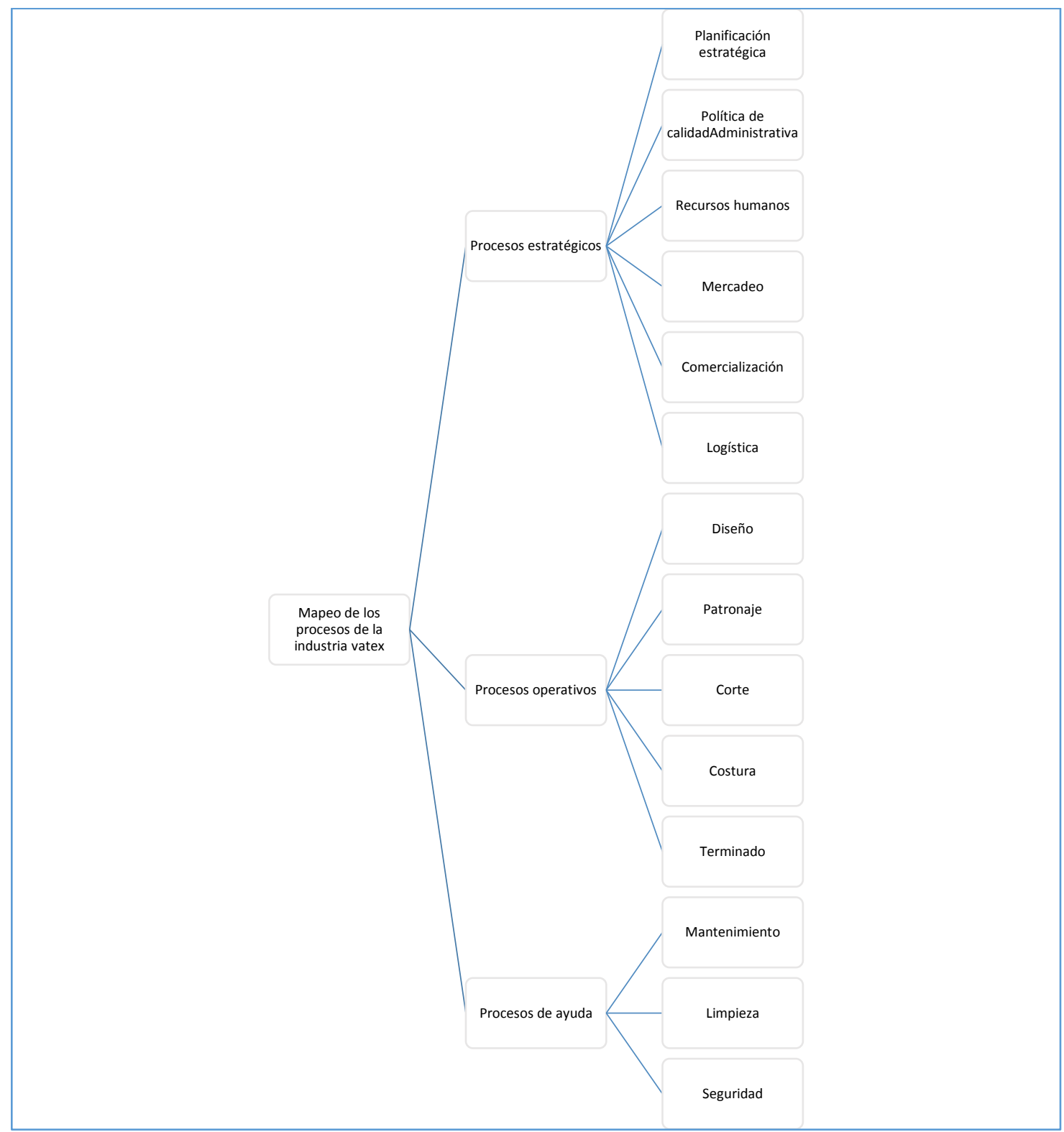

Fuente. Vatex

Los procesos estratégicos se encuentran definidos para ser observados y realizados por parte de la alta gerencia. Estos procesos están orientados al cumplimiento del objetivo estratégico de la empresa, bajo el monitoreo continuo para dar cumplimiento al plan y las políticas institucionales. 
Los procesos operativos parten desde el diseño de los productos hasta su etapa final de producción que es el terminado.

Los procesos de apoyo abarcan actividades que de una u otra forma contribuyen al normal desarrollo de las operaciones empresariales, tales como: servicios de mantenimiento, limpieza, seguridad y bodegaje.

Con la aplicación del modelo se desarrolló el esquema, producto del estudio de cada una de las fases, en la Tabla 3 se puede observar los procesos y las actividades que cada uno desarrolla.

Tabla 3. Cuadro de resumen de actividades

\begin{tabular}{|c|c|}
\hline Procesos Relevantes & $\begin{array}{l}\text { Número de } \\
\text { actividades }\end{array}$ \\
\hline Procesos Operativos: & 34 \\
\hline Diseño & 10 \\
\hline Patronaje & 7 \\
\hline Corte & $\underline{5}$ \\
\hline Costura & 4 \\
\hline Terminados & 8 \\
\hline Procesos Estratégicos: & 67 \\
\hline Definición y seguimiento de la estrategia & 10 \\
\hline Gestión de litigios & 5 \\
\hline Gestión tributaria & 3 \\
\hline Gestión contable & 10 \\
\hline Gestión financiera & 5 \\
\hline Gestión de importaciones & 5 \\
\hline Reclutamiento de personal & 10 \\
\hline Evaluación de personal & 10 \\
\hline $\begin{array}{l}\text { Cumplimiento de reglamento de salud y seguridad } \\
\text { ocupacional }\end{array}$ & 3 \\
\hline Distribución de mercadería & 3 \\
\hline Gestión de inventarios & 3 \\
\hline Procesos de apoyo & $\underline{16}$ \\
\hline Procesos técnicos & 3 \\
\hline Procesos de seguridad & 3 \\
\hline Procesos de bodegaje & 10 \\
\hline Total & 117 \\
\hline
\end{tabular}

Fuente. Vatex 
Detallado los procesos operativos se establece el flujograma del proceso productivo:

Figura 5. Flujograma del proceso de producción.

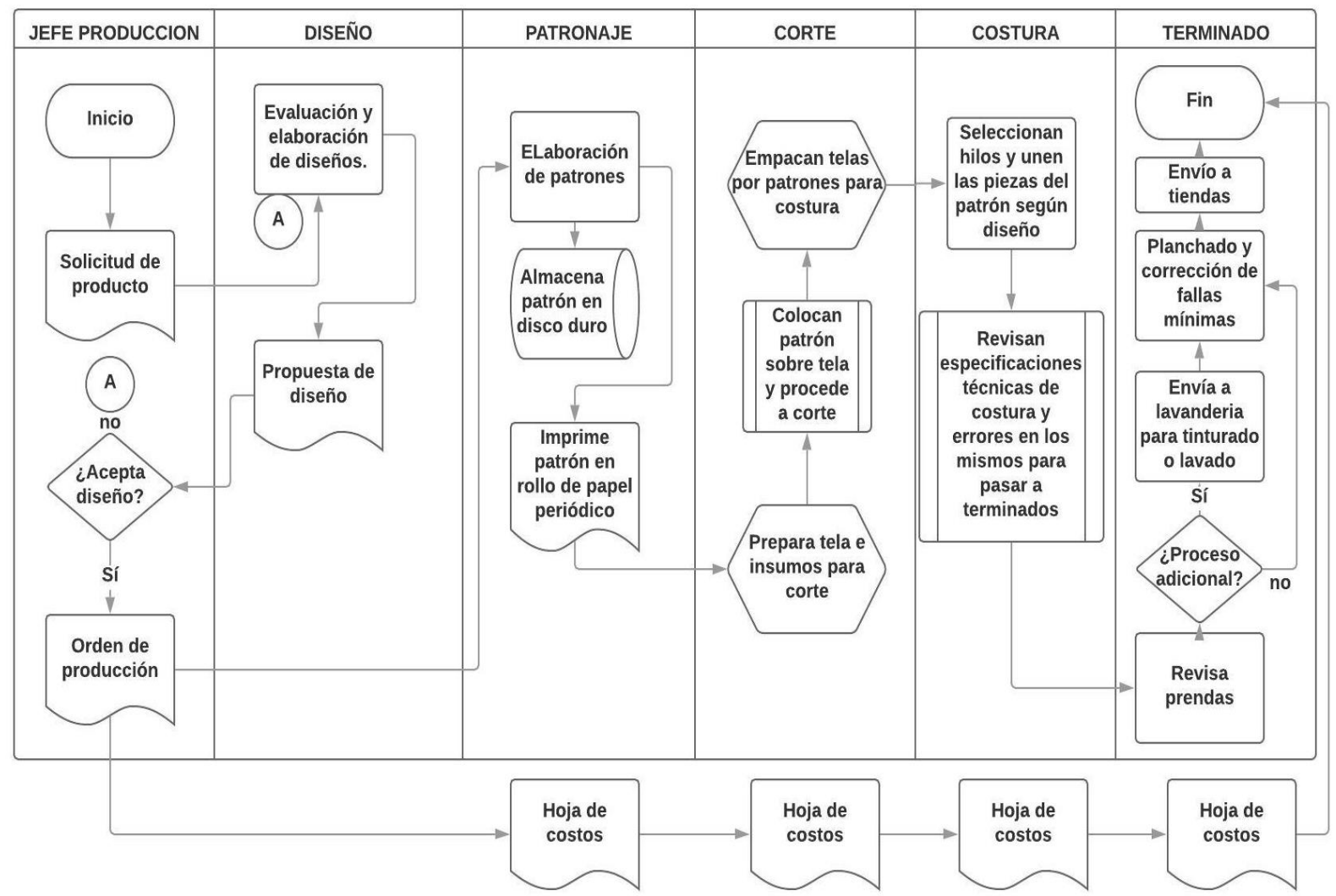

Fuente. Vatex

Este flujograma permite realzar la importancia de contar con un proceso definido, en el que se pueden ver las actividades que desarrolla cada área desde el diseño hasta su empaque y envío a las diferentes sucursales. Para la visualización de los costos que mantiene la empresa de forma mensual, se recolectó la información que a continuación se presenta en la figura 5, en el mismo contexto se propone prorratear el total de los costos indirectos para el número de horas mensuales que se producen en la empresa.

Para la presente investigación se analizaron los costos que incurren en la elaboración de la línea denominada por la empresa como básicos, estos son camisetas y pantalones jean, siendo estos los objetos del costo. 
Tabla 4. Elementos del CIF de prendas de vestir

\begin{tabular}{|l|r|}
\hline \multicolumn{1}{|c|}{ CIF } & $\mathbf{5 . 9 6 0 , 5 0}$ \\
\hline Insumos (hilos, entretela, botones, cordon, elás & 869,50 \\
\hline Energia eléctrica & 474,40 \\
\hline Agua & 26,52 \\
\hline Depreciación de maquinaria de producción & 618,12 \\
\hline Mantenimiento maquinaria & 130,53 \\
\hline Arriendo & $1.402,50$ \\
\hline Seguridad y monitoreo & 52,39 \\
\hline Seguro & 314,74 \\
\hline Suinistros fábrica & $1.137,20$ \\
\hline Suministros diseño & 545,93 \\
\hline Repuestos y herramientas & 388,67 \\
\hline TOTAL & $\mathbf{5 . 9 6 0 , 5 0}$ \\
\hline
\end{tabular}

Fuente. Vatex

Una vez que se han identificado los cotos de producción, incluidos mano de obra, materia prima y costos indirectos, se procede a distribuir los recursos de acuerdo a los tiempos calculados para cada actividad, consecuentemente se determina el costo unitario de los productos elaborados (tabla 5).

Tabla 5. Asignación de recursos a actividades

\begin{tabular}{|c|c|c|c|c|c|}
\hline \multirow{2}{*}{ Procesos Relevantes } & \multirow{2}{*}{ Inductor } & \multicolumn{2}{|c|}{ Tiempo } & \multicolumn{2}{|c|}{ Asignación de costos } \\
\hline & & Camisetas & Pantalones & Camisetas & Pantalones \\
\hline Procesos Operativos: & & & & 930,65 & 981,45 \\
\hline Diseño & Hora de mano de obra & 1,00 & 1,00 & 33,87 & 33,87 \\
\hline Patronaje & Hora de mano de obra & 3,08 & 3,08 & 104,31 & 104,31 \\
\hline Corte & Hora de mano de obra & 3,75 & 3,75 & 127,00 & 127,00 \\
\hline Costura & Hora de mano de obra & 16,65 & 18,75 & 563,88 & 635,00 \\
\hline Terminados & Hora de mano de obra & 3,00 & 2,40 & 101,60 & 81,28 \\
\hline Procesos Estratégicos: & & & & 19,30 & 19,30 \\
\hline Definición y seguimien to de la estrategia & Hora de mano de obra & 0,05 & 0,05 & 1,69 & 1,69 \\
\hline Gestión de litigios & Hora de mano de obra & 0,05 & 0,05 & 1,69 & 1,69 \\
\hline Gestión tributaria & Hora de mano de obra & 0,05 & 0,05 & 1,69 & 1,69 \\
\hline Gestión contable & Hora de mano de obra & 0,05 & 0,05 & 1,69 & 1,69 \\
\hline Gestión in anciera & Hora de mano de obra & 0,05 & 0,05 & 1,69 & 1,69 \\
\hline Gestión de importaciones & Hora de mano de obra & 0,05 & 0,05 & 1,69 & 1,69 \\
\hline Recluta miento de personal & Hora de mano de obra & 0,05 & 0,05 & 1,69 & 1,69 \\
\hline Evalua ción de personal & Hora de mano de obra & 0,05 & 0,05 & 1,69 & 1,69 \\
\hline $\begin{array}{l}\text { Cumplimiento de regla mento de salud y } \\
\text { segurid ad ocupacional }\end{array}$ & Hora de mano de obra & 0,02 & 0,02 & 0,68 & 0,68 \\
\hline Distribución de mer ca dería & Hora de mano de obra & 0,10 & 0,10 & 3,39 & 3,39 \\
\hline Gestión de inventarios & Hora de mano de obra & 0,05 & 0,05 & 1,69 & 1,69 \\
\hline Procesos de apoyo & & & & 2,37 & 2,37 \\
\hline Procesos técnicos & Hora de mano de obra & 0,05 & 0,05 & 1,69 & 1,69 \\
\hline Procesos de seguridad & Hora de mano de obra & 0,01 & 0,01 & 0,34 & 0,34 \\
\hline Procesos de bodegaje & Hora de mano de obra & 0,01 & 0,01 & 0,34 & 0,34 \\
\hline Total & & 28,12 & 29,62 & 952,32 & $1.003,12$ \\
\hline
\end{tabular}


Para el análisis de la rentabilidad de las prendas de vestir, se realizó una comparación entre los costos previamente establecidos por la empresa y los datos analizados técnicamente (aplicación del sistema $\mathrm{ABC} / \mathrm{ABM}$ ), dando como resultado la información presentada en la tabla 6:

Tabla 6. Margen de rentabilidad camisetas.

\begin{tabular}{|c|c|c|}
\hline \multicolumn{3}{|l|}{ Camisetas } \\
\hline & Empírico & $\mathrm{ABC} / \mathrm{ABM}$ \\
\hline Costo & 7,48 & 9,00 \\
\hline Precio de venta & 13,95 & 13,95 \\
\hline Utilidad Bruta & 6,47 & 4,95 \\
\hline CIF individual (cargado al gasto) & 4,21 & 0 \\
\hline Utilidad Neta & 2,26 & 4,95 \\
\hline
\end{tabular}

Con la aplicación del sistema ABC/ABM, expuesto en la tabla 6, se evidencia que existe una mayor utilidad neta, esto debido a que ya se toma en consideración como costo los valores atribuidos como gastos.

Tabla 7. Margen de rentabilidad pantalones.

\begin{tabular}{|c|c|c|}
\hline \multicolumn{3}{|c|}{ Pantalones } \\
\hline & Empírico & $A B C / A B M$ \\
\hline Costo & 15,75 & 27,24 \\
\hline Precio de venta & 32,95 & 32,95 \\
\hline Utilidad & 17,20 & 5,71 \\
\hline CIF individual (cargado al gasto) & 12,47 & 0 \\
\hline Utilidad Neta & 4,73 & 5,71 \\
\hline
\end{tabular}

En el cálculo que antecede se puede visualizar un incremento en los costos incurridos para la producción de pantalones, a través del uso de costos $\mathrm{ABC} / \mathrm{ABM}$ en contraste con el método empírico, no obstante, se evidencia una mayor utilidad obtenida, siendo, por tanto, favorable su aplicación en este tipo de empresas.

Haciendo un análisis de las deudas adquiridas para la adquisición de materia prima e insumos para la producción y también las deudas financieras y su costo financiero, se aplica el WACC como indicador para medir el riesgo de la empresa frente a inversiones nuevas. 


$$
\mathrm{WACC}=\frac{100.000}{150.000} \times 20,76 \%+\frac{50.000}{150.000} \times 0
$$

En dónde:

\section{$\mathrm{WACC}=13,84 \%$}

Df: $100.000,00$

Cdf: $20,76 \%$

Fp: $50.000,00$

Cfp: 0

Rt: $150.000,00$

Este resultado da a conocer que el riesgo de realizar inversiones en esta empresa es bajo considerando que la entidad no cuenta con préstamos bancarios para financiar la producción de las prendas de vestir, sino que lo realiza con recursos propios, esto genera un escenario más atractivo para los futuros inversionistas.

\section{Conclusiones.}

- La industrialización de los productos y servicios, así como su diversificación, hacen necesaria la aplicación de métodos que puedan ser utilizados para el correcto control de asignaciones de costos, generando una guía que ayude a tomar decisiones acertadas y fijar precios de manera técnica y analítica a los productos que se ofertan al consumidor final.

- La generación de resultados claros, son la base para establecer un modelo de gestión de costos y con la aplicación del mismo, permitir un análisis de los resultados; este modelo se considera como una herramienta de gran ayuda para la alta gerencia en la toma de decisiones

- La información que se obtuvo con la aplicación de la encuesta, permite evidenciar que existe ausencia de un proceso de costos definido por la empresa y la información para análisis de razones no es confiable.

- Se determinó que la aplicación del sistema de costos ABC/ABM permite mejorar la rentabilidad, en el caso de la producción de camisetas, la rentabilidad sube a un 55\% y de pantalones un $21 \%$. Esto se debe a que los costos indirectos de fabricación forman parte del producto, generando una mejor base para la toma de decisiones, correcta asignación de precios de venta y visualización de que productos son los que ayudan a mantener la línea de negocio.

- La aplicación de un modelo de gestión de costos ABC/ABM es posible en las productoras textiles, permitiendo obtener información real para la evaluación de las inversiones y su riesgo; además, ayuda a los inversionistas a visualizar lo atractivo de esta empresa. 


\section{Referencias bibliográficas.}

Adame, R. (2000). Costeo basado en actividades (ABC) Conceptos teóricos y metodología de implementación (tesis de maestría). Universidad Autónoma de Nuevo León, Nuevo León, México.

Aragón, A. y Rubio, A. (2006). Factores Explicativos del éxito competitivo: el caso de las Pymes del Estado de Veracruz. Revista CyA, volumen 64-1, 35-69. Recuperado de: http://www.cya.unam.mx/index.php/cya/article/view/568

Arrellano, O., Quishpe, G., Ayaviri, D., y Escobar, F. (2017). Estudio de la aplicación del método de costos ABC en las Mypes del Ecuador. Revista de Investigaciones Altoandinas, volumen 19, 33-46. Recuperado de: http://www.scielo.org.pe/scielo.php?script=sci_arttext\&pid=S231329572017000100004

Cano, A. (2013). Contabilidad Gerencial y presupuestaria aplicada a las ciencias económicas, administrativas y contables. Bogotá, Colombia: Ediciones de la U.

Cantero, H., y Leyva, E. (2016). La rentabilidad económica, un factor para alcanzar la eficiencia empresarial. Ciencias Holguín, volumen 22, 1-17. Recuperado de: http://www.redalyc.org/articulo.oa?id=181548029004

Chacón, G. (2007). La contabilidad de costos, los sistemas de control de gestión y la rentabilidad empresarial. Actualidad Contable Faces, volumen 10, 29-45. Recuperado de: http://www.redalyc.org/pdf/257/25701504.pdf

Cuervo, J., Osorio, J., y Duque, M. (2013). Costeo basado en actividades ABC Gestión basada en actividades ABM (Vol. 2). Bogotá, Colombia: Ecoe Ediciones.

Eras, R., Burgos, J., \& Lalangui, M. (2016). Contabilidad de costos (Primera ed.). Machala: UTMACH.

Horngren, C., Datar, S., y Rajan, M. (2012). Contabilidad de costos. Un enfoque gerencial. Naucalpán de Juárez, México: Pearson Educación.

Kaplan, R., \& Anderson, S. (2008). Costes basados en el tiempo invertido por actividad. Barcelona: Deusto S.A. Ediciones .

Navarro, F. (24 de Septiembre de 1995). www.observatorio-iberoamericano.org. Obtenido de Obervatorio iberoamericano: http://www.observatorioiberoamericano.org/paises/Spain/Art\%C3\%ADculos\%20diversos\%20sobre\%20 Contabilidad\%20de\%20Gesti\%C3\%B3n/ABM\%20-\%20Navarro.htm

Maldonado, S. (2003). La Información Contable en la Gestión Empresarial: Una Reflexión a partir de la Experiencia en Consultoría de la Pontificia Universidad Javeriana. Revista Economía, gestión y desarrollo, volumen 1, 107-125. Recuperado de: http://revistaeconomia.puj.edu.co/html/articulos/Numero_1/art5.pdf

Molina, A., \& Morillo, M. (2000). Sistemas de acumulación de costos para empresas de servicio. Revista Actualidad Contable, 48-55.

Morillo, M. (2001). Rentabilidad financiera y reducción de costos. Actualidad contable FACES, 36.

Pérez, O., y Tapanes, Y. (2009). Origen del sistema de gestión y costos basado en actividades (ABC/ABM). Revista avanzada científica, volumen 12, páginas. Recuperado de: https://dialnet.unirioja.es/servlet/articulo?codigo=5074421 
Polimeni, R., Fabozzi, F., Adelberg, A., y Kole, M. (Ed. tercera) (1997). Contabilidad de costos. Santafé de Bogotá, Colombia: McGRAW-HILL.

Rincon, C. (2012). Auditoría de costos. Bogotá, Colombia: Ecoe Ediciones.

Robles, C. (2012). Fundamentos de administración financiera. Viveros de la Loma, Tlalnepantla, México: Red Tercer Milenio S.C.

Sánchez, J. (2002). Análisis de la rentabilidad de la empresa. Universidad de Zaragoza. Recuperado de http://www.5campus.com/leccion/anarenta

Santiesteban, E., Fuentes, V., Leyva, E., Lozada, D., y Cantero, H. (2011). Análisis de la rentabilidad económica. Tecnología propuesta para incrementar la eficiencia empresarial. La Habana, Cuba: Editorial Universitaria.

Shim, J. y Siegel, J. (1988). Administración Financiera. Bogotá, Colombia: Editorial McGrow-Hill Latinoamericana S.A. 


\section{PARA CITAR EL ARTÍCULO INDEXADO.}

González Montaño, H., Narváez Zurita, C., Lituma Yascaribay, M., \& Erazo Álvarez, J. (2019). Sistema de gestión de costos ABC/ABM para la industria de textiles. Caso empresa Vatex. Visionario Digital, 3(2.1.), 260-283.

https://doi.org/10.33262/visionariodigital.v3i2.1.555

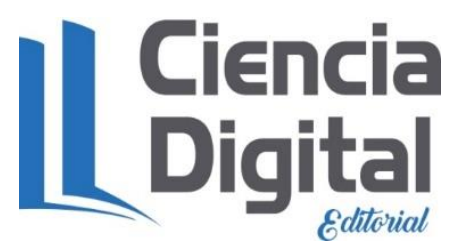

El artículo que se publica es de exclusiva responsabilidad de los autores y no necesariamente reflejan el pensamiento de la Revista Ciencia Digital.

El artículo queda en propiedad de la revista y, por tanto, su publicación parcial y/o total en otro medio tiene que ser autorizado por el director de la Revista Ciencia Digital.
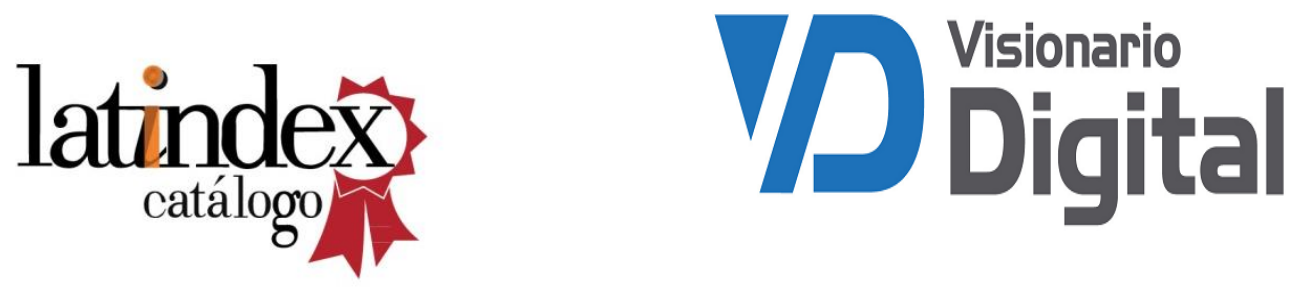